Pacific Journal of Mathematics

AN ADJUNCTION THEOREM FOR LOCALLY 


\title{
AN ADJUNCTION THEOREM FOR LOCALLY EQUICONNECTED SPACES
}

\author{
Eldon Dyer and S. Eilenberg
}

The locally equiconnected spaces (LEC spaces) can be characterized as the spaces $X$ with the property that if $f_{0}, f_{1}: Z \rightarrow X$ are mappings which are "sufficiently close together" and which agree on a subspace $A$ of $Z$, then $f_{0}$ is homotopic to $f_{1}$ relative to $A\left(f_{0} \cong f_{1}\right.$ rel $\left.A\right)$; i.e., there is a morphism $F: Z \times I \rightarrow X$ with $F\left|Z \times 0=f_{0}, F\right| Z \times 1=f_{1}$ and $F(a, t)=f_{0} a$ for all $a \in A$ and $t \in I$.

The notion of "close" is measured by a morphism $\varphi: X \times$ $X \rightarrow I$ with $\varphi\left(x, x^{\prime}\right)=0$ if and only if $x=x^{\prime}$. We then require that $\varphi\left(f_{0} \xi, f_{1} \xi\right)<1$ for all $\xi \in Z$ implies that $f_{0} \cong f_{1}$ rel $A$.

There is a universal test pair $(u, D)$ : let $u=\varphi^{-1}[0,1>$ and $D=\varphi^{-1} 0$. Let $f_{0}$ and $f_{1}$ be the restrictions to $u$ of the projections $X \times X \rightarrow X$ onto the first and second coordinates. Then $f_{0}$ and $f_{1}$ agree precisely on $D$. A homotopy $f_{0} \cong f_{1}$ rel $D$ exists if and only if $D$ is a strong deformation retract of $u$ in $X \times X$.

We note two things. First, the existence of a homotopy $f_{0} \cong f_{1}$ rel $D$ implies the existence of the homotopies in the general case described in the first paragraph. Second, the existence of a map $\varphi$ and homotopy $f_{0} \cong f_{1}$ rel $D$ is equivalent to the diagonal map

$$
\Delta: X \rightarrow X \times X
$$

being a cofibration.

We say more on this point below.

The class of LEC spaces has been the subject of considerable investigation (see Dugundji [1] for background) and such spaces have a number of convenient homotopy theoretic properties. To establish contact with a more familiar class of spaces, we recall that every metric absolute neighborhood retract (ANR) is LEC and that every finite dimensional metric LEC space is an ANR. (See [1]).

One of the beautiful results on ANR's is the Whitehead Adjunction Theorem for compact ANR's [7]. This has been the subject of several generalizations [3] and [4]. The object of this paper is to present an adjunction theorem for LEC spaces analogous to Whitehead's but with no restriction on the LEC spaces involved. A corollary is that every cell-complex is locally equiconnected.

I. Preliminaries. We do not wish to be too specific about the category of spaces under consideration. Specific categories for which 
all of the ensuing arguments are valid are the category of compactly generated Hausdorff spaces [6] (which includes metrizable spaces and locally compact Hausdorff spaces) and the category of quasi-topological spaces [5]. A third category in which they are valid is the category of compactly generated weakly Hausdorff spaces; i.e., spaces in which the topology is generated by continuous maps of compact Hausdorff spaces; there appears to be no material in print on this category.

The terms continuous mapping and morphism will be used interchangeably. A morphism $f: A \rightarrow X$ is an injection provided it is oneto-one into and has the property that a sufficient condition for a function $g: Y \rightarrow A$ to be a continuous mapping is that the composition $f g$ be one. If $f$ is an inclusion and an injection, then $A$ is said to have the subspace topology or the induced topology. Dually, a morphism $p: X \rightarrow B$ is a projection provided it is onto and has the property that a sufficient condition for a function $q: B \rightarrow Y$ to be a continuous mapping is that the composition $q p$ be one. In this case $B$ is said to have the quotient or decomposition topology determined by $p$.

A subset $A$ of the space $X$ has a halo in $X$ if there is a morphism $q: X \rightarrow I$ such that $A=q^{-1} 0$. Such a morphism is called a haloing function for $A$. Note that only $G_{\delta}$ closed subsets can have haloes. If, in addition to having a halo in $X, A$ is also a retract of $X$, then $A$ is said to be a halo retract of $X$.

A morphism $k: X \rightarrow I$ is a numeration of an open set $U$ in $X$ provided $U=X-k^{-1} 1$. An open set $U$ is numerable if there exists a numeration of it.

In each of the above categories, there are arbitrary function spaces $M(X, Y)$ in which the exponential law is valid and the evaluation map is a morphism.

For a space $X$ the path space $P X$ is the subspace of $M\left(R^{-}, X\right) \times$ $R^{+}$, where $R^{+}$is the half-line of nonnegative real numbers, of all pairs $(\alpha, l)$ with $\alpha(t)=\alpha(l)$ for all $t \geqq l$. A path is a point $(\alpha, l)$ of $P X$; the number $l$ is said to be the length of the path $(\alpha, l)$. We define two morphisms

$$
\eta_{0}: P X \longrightarrow X
$$

and

$$
\eta_{\tau}: P X \longrightarrow X
$$

by $\eta_{0}(\alpha, l)=\alpha 0$ and $\eta_{\tau}(\alpha, l)=\alpha l$.

We next state several lemmas and propositions to be used later. We omit proofs of the more routine of these.

Lemma I.1. Let $\varphi: X \rightarrow I$ be a morphism and $\psi: X \rightarrow I$ be a func- 
tion such that

(i) $\psi \mid u$ is a morphism, $\left.u=\varphi^{-1}<0,1\right]$, and

(ii) $\psi \leqq \varphi$.

Then \& is a morphism.

Lemma I.2. Let $\varphi: X \rightarrow I, \psi: Y \rightarrow I$, and $h: X \times I \rightarrow Z$ be morphisms such that

$$
h(x, t) \text { is independent of } t \text { if } \varphi(x)=0 .
$$

Let $W$ be the subspace of $X \times Y \times I$

$$
W=\{x, y, t \in X \times Y \times I \mid t \psi y \leqq \varphi x\}
$$

and $l: W \rightarrow Z$ be the function

$$
l(x, y, t)=\left\{\begin{array}{lll}
h(x, t \psi(y) / \varphi x) & \text { if } & \varphi x \neq 0 \\
h(x, 0) & \text { if } & \varphi x=0 .
\end{array}\right.
$$

Then $l$ is a morphism.

Proof. Let $\gamma: C \rightarrow W$ be a morphism, where $C$ is compact Hausdorff. The map $\gamma$ is given by coordinates

$$
\gamma_{1}: C \longrightarrow X, \gamma_{2}: C \longrightarrow Y \text { and } \gamma_{3}: C \longrightarrow I \text {. }
$$

The definition of $W$ requires that

$$
\gamma_{3} \cdot\left(\psi \gamma_{2}\right) \leqq \varphi \gamma_{1}
$$

Let $\alpha: C \times I \rightarrow I \times I$ be defined by $\alpha(c, t)=\left(t \varphi \gamma_{1} c,, \gamma_{3} c \cdot \psi \gamma_{2} c\right)$ and $D=$ $\alpha^{-1}\{\Delta \cup 0 \times I\}$. Since $\alpha$ is continuous, $D$ is compact; and so, $\pi_{1}: D \rightarrow C$ is a projection since it is onto. The composition

$$
D \stackrel{\pi_{1}}{\longrightarrow} C \stackrel{\gamma}{\longrightarrow} W \stackrel{l}{\longrightarrow} Z
$$

is

$$
\begin{aligned}
l \gamma \pi_{1}(c, t) & =l \gamma c=l\left(\gamma_{1} c, \gamma_{2} c, \gamma_{3} c\right) \\
& = \begin{cases}h\left(\gamma_{1} c, \gamma_{3} c \frac{\psi \gamma_{2} c}{\varphi \gamma_{1} c}\right) & \text { if } \varphi \gamma_{1} c \neq 0 \\
h\left(\gamma_{1} c, 0\right) & \text { if } \varphi \gamma_{1} c=0\end{cases} \\
& = \begin{cases}h\left(\gamma_{1} c, t\right) & \text { if } \varphi \gamma_{1} c \neq 0 \\
h\left(\gamma_{1} c, 0\right) & \text { if } \varphi \gamma_{1} c=0\end{cases} \\
& =h\left(\gamma_{1} c, t\right),
\end{aligned}
$$

which is a morphism. Since $\pi_{1}: D \rightarrow C$ is a projection, this implies $l \gamma$ is a morphism. But since this is true for every morphism $\gamma: C \rightarrow W$, 
it follows that $l$ is a morphism.

Lemma I.3. For C compact Hausdorff, the function

$$
\text { sup: } M(C, R) \longrightarrow R
$$

defined by $\sup f=\sup \{f c \mid c \in C\}$ is a morphism.

Proposition I.4. The function

$$
j: P Y \longrightarrow M(I, Y) \times R^{+}
$$

defined by $j(\alpha, l)=(\hat{\alpha}, l)$, where $\hat{\alpha} t=\alpha(t \cdot l)$, is an injection.

Proof. It is clear that $j$ is a morphism and is a set-theoretic injection. We have only to show that if $f: C \rightarrow P Y$ is a function, where $C$ is compact Hausdorff, such that $j f$ is a morphism, then $f$ is a morphism.

We assume $j f$ is a morphism. This implies both $\pi_{1} j f$ and $\pi_{2} j f$ are morphisms.

If $\sup \pi_{2} j f=0$, then $\operatorname{Im} f$ is contained in the subspace of $P Y$ of paths of length 0 . This subspace is homeomorphic to $Y$ and is mapped by $\pi_{1} j$ homeomorphically onto the subspace of constant paths in $M(I, Y)$. Thus, since $\pi_{1} j f$ is a morphism, so is $f$.

Otherwise, let

$$
\varphi: C \longrightarrow I \text { be } \varphi c=\pi_{2} j f c / \sup \pi_{2} j f .
$$

Let $D \subset C \times I$ be $\{c, t \mid t \leqq \varphi c\}$. The composition

$$
D \longrightarrow C \times I \stackrel{\tilde{f}}{\longrightarrow} Y
$$

is a morphism, where $\widetilde{f}(c, t)=f c\left(t \pi_{2} j f c\right)$ is adjoint to $\pi_{1} j f$ and the first morphism is defined by $(c, t) \rightarrow(c, t / \varphi c)$.

Let $E$ be the subspace of $C \times R^{+}$of all pairs $(c, s)$ with $s \leqq \pi_{2} j f c$ and map $E$ to $D$ by sending $(c, s)$ to $\left(c, s / \sup \pi_{2} j f\right)$. Finally, define $\gamma: C \times R^{+} \rightarrow E$ by

$$
\gamma(c, u)=\left\{\begin{array}{lll}
c, u, & \text { if } \quad u \leqq \pi_{2} j f c \\
c, \pi_{2} j f c, & \text { if } \quad \pi_{2} j f c \leqq u
\end{array}\right.
$$

The composition

$$
C \times R^{+} \stackrel{r}{\longrightarrow} E \longrightarrow D \longrightarrow Y
$$

is a morphism taking $(c, u)$ to $(f c)(u)$. Its adjoint is $\pi_{1} f: C \rightarrow M\left(R^{+}, Y\right)$, which is thus a morphism. Since $\pi_{2} f$ is the morphism $\pi_{2} j f$, it follows that $f$ is a morphism. 
Lemma I.5. Let $g: X \rightarrow P Y$ and $f: X \rightarrow R^{+}$be morphisms such that $f^{-1} 0 \subset\left(\pi_{2} g\right)^{-1} 0$. Then the function

$$
\hat{g}: X \longrightarrow P Y
$$

defined by $\hat{g} x(t \cdot f x)=g x\left(t \cdot \pi_{2} g x\right), t \in I$, with $\pi_{2} \hat{g}=f$, is a morphism.

Proof. $\hat{g}$ is well-defined since $f^{-1} 0 \subset\left(\pi_{2} g\right)^{-1} 0 . \quad \pi_{1} j \hat{g}=\pi_{1} j g$ and $\pi_{2} j \hat{g}=f$ are morphisms; thus, $j \widehat{g}$ is a morphism. Since $j$ is an injection, it follows that $\hat{g}$ is a morphism.

The following companion theorems are proved by variants of a method of G. S. Young in [8]. We recall that a morphism $f: A \rightarrow X$ is a cofibration provided the diagram

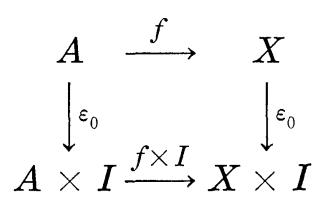

is a weak pushout; i.e., given $F: A \times I \rightarrow Y$ and $g: X \rightarrow Y$ with $F \varepsilon_{0}=$ $g f$, there exists a $G: X \times I \rightarrow Y$ (not necessary unique) such that $G \varepsilon_{0}=$ $g$ and $G f \times I=F$.

Theorem I.6. Every cofibration $f: A \rightarrow X$ is an injection of $A$ on a closed subset of $X$ which has a halo.

Theorem I.7. Let $A$ be a closed subset of $X$ and $i: A \rightarrow X$ be the injection. Then the following properties are equivalent:

(i) $i$ is a cofibration.

(ii) $A \times I \cup X \times 0$ is a retract of $X \times I$,

(iii) there exist a halo $U$ of $A$ and a morphism

$$
h: X \times I \longrightarrow X
$$

such that

$$
h(x, 0)=x, h(a, t)=a, h(u, 1) \in A
$$

for $x \in X, a \in A, t \in I$, and $u \in U$,

(iv) there exist a halo $V$ of $A$ and a morphism

$$
h: V \times I \longrightarrow X
$$

such that

$$
h(v, 0)=v, h(a, t)=a, h(v, 1) \in A
$$

for $v \in V, a \in A$, and $t \in I$, and 
(v) there exist a haloing function $\varphi$ for $A$ in $X$ and a morphism

$$
h: V \longrightarrow P X, \text { where } V=\varphi^{-1}[0,1>\text {, }
$$

such that

$$
\eta_{0} h=1_{v}, \eta_{\tau} h(v) \subset A, l h=\varphi \mid V
$$

II. Properties of LEC spaces. We shall define a space $X$ to be locally equiconnected (abbreviated LEC) provided the diagonal map

$$
\Delta: X \longrightarrow X \times X
$$

is a cofibration. This is in agreement with earlier usage [1].

THEOREM II.1. The space $X$ is LEC if and only if there exist morphisms

$$
\begin{aligned}
& k: X \times X \longrightarrow I \\
& g: V \longrightarrow P X, \text { for } V=k^{-1}[0,1>,
\end{aligned}
$$

such that

(i) $k^{-1} 0=D$, the diagonal in $X \times X$ and

(ii) $\eta_{0} g=\pi_{1}\left|V, \eta_{\tau} g=\pi_{2}\right| V$ and $l g=k$, where $\pi_{1}$ and $\pi_{2}$ are the projections of $X \times X$ onto its first and second factors and for a path $\alpha, l \alpha$ is the length of the path.

We shall refer to a pair of morphisms $(k, g)$ having the properties of this theorem as LEC-data for $X$.

Proof of Theorem. If $\Delta: X \rightarrow X \times X$ is a cofibration, then by condition (v) of Theorem I.6 there exist morphisms

$$
\begin{aligned}
& \varphi: X \times X \longrightarrow I, \text { with } D=\varphi^{-1} 0, \text { and } \\
& h: V \longrightarrow P(X \times X), \text { where } V=\varphi^{-1}[0,1>,
\end{aligned}
$$

such that $h v$ is a path from $v$ to $D$ of length $\varphi v$. Let $k=2 \varphi$ and define

$$
g: V \longrightarrow P X
$$

to be $g=\pi_{1} h-\pi_{2} h$. Since $h v 0=v, g v$ is a path in $X$ from $\pi_{1} v$ to $\pi_{2} v$. Its length is $2 \varphi v=k v$.

Suppose $(k, g)$ are LEC-data for $X$. Let $\varphi=k$ and define

$$
h: V \longrightarrow P(X \times X), V=\varphi^{-1}[0,1>,
$$

to be $\left(g, \pi_{2}\right)$. Since $g v$ is a path from $\pi_{1} v$ to $\pi_{2} v, h v$ is a path from 
$v$ to $\left(\pi_{2} v, \pi_{2} v\right) \in D$. Its length is $k v=\varphi v$

TheOREM II.2. If $X_{1}, X_{2}, \cdots, X_{n}$ are LEC, then $X=\prod_{i=1}^{n} X_{i}$ is LEC.

Proof. Since $\Delta_{i}: X_{i} \rightarrow X_{i} \times X_{i}$ is a cofibration for each $i$,

$$
\prod_{i} \Delta_{i}: X \longrightarrow \prod_{i}\left(X_{i} \times X_{i}\right)
$$

is a cofibration. This morphism composed with the twisting homeomorphism

$$
T: \prod_{i}\left(X_{i} \times X_{i}\right) \longrightarrow X \times X
$$

is the diagonal map for $X$. Thus, $\Delta_{X}$ is a cofibration and $X$ is LEC.

THEOREM II.3. A LEC space $X$ can be covered by numerated open sets contractible in $X$. Also, $X$ is Hausdorff.

Proof. Let $k, g$ be LEC-data for $X$ and $V=k^{-1}[0,1>$.

For $x \in X$, let $V_{x}=\{y \in X \mid(x, y) \in V\}$. Define $k_{x}: X \rightarrow I$ by $k_{x}(y)=$ $k(x, y)$. Then the open set $V_{x}$ is numerated by $k_{x}$. Define

$$
C_{x}: V_{x} \times I \longrightarrow X
$$

by $C_{x}(y, t)=g(x, y) t$. This is a deformation in $X$ of $V_{x}$ into $x$.

As in $\S 4$, number 4 of page 253 of [2], it follows that if $X$ is also compact, then it is metrizable.

For $x \neq y$, let $S_{x}=\left\{\xi \in X \mid k_{x}(\xi)<k_{y}(\xi)\right\}$ and $S_{y}=\left\{\xi \in X \mid k_{y}(\xi)<k_{x}(\xi)\right\}$. The sets $S_{x}$ and $S_{y}$ are disjoint open sets in $X$ containing $x$ and $y$, respectively.

THEOREM II.4. In a LEC space the path components of a numerated open set are open.

Proof. Let $k, g$ be LEC-data for $X$ and $V_{x}$ be as in the previous proof.

For $\psi^{\prime}: X \rightarrow I, U=$ Support $\psi$ and $x \in U$, define

$$
\bar{\psi}: V_{x} \longrightarrow I
$$

by $\bar{\psi} y=\inf \{\psi g(x, y) t \mid t \in I\}$. Then $0<\psi(x)=\bar{\psi}(x)$. If $0<\bar{\psi} y$, then $g(x, y) I \subset U$. Thus, the path component of $x$ in $U$ contains the support of $\bar{\psi}$, which is open.

Corollary II.5. If $X$ is LEC, the decomposition space $\Pi X$ of 
path components of $X$ is discrete.

Proof. The function 1: $X \rightarrow I$ has support $X$. By the previous theorem the path components of $X$ are open.

For a compact space $C$, a closed subset $A$ of $C$ and a map $\varphi: A \rightarrow X$, denote by $(C, X, \varphi)$ the subspace of the space $M(C, X)$ of maps of $C$ into $X$ of those functions $f: C \rightarrow X$ such that $f \mid A=\varphi$.

Theorem II.6. If $X$ is LEC, then so is $(C, X, \varphi)$.

Proof. Let $k, g$ be LEC-data for $X$. For $f, f^{\prime} \in M(C, X)$, let

$$
\bar{k}\left(f, f^{\prime}\right)=\sup \left\{k\left(f c, f^{\prime} c\right) \mid c \in C\right\} .
$$

The function $\bar{k}$ is a morphism since it is the composition of the morphism

$$
\text { sup: } M(C, I) \longrightarrow I
$$

with the adjoint of the composition

$$
\begin{aligned}
C \times M(C, X) \times M(C, X) \stackrel{\Delta_{\mathrm{C}} \times 1}{\longrightarrow} C \times C \times M(C, X) \times M(C, X) \\
\stackrel{1 \times T \times 1}{\longrightarrow} C \times M(C, X) \times C \times M(C, X) \\
\stackrel{e \times e}{\longrightarrow} X \times X \stackrel{k}{\longrightarrow} I,
\end{aligned}
$$

where $e: C \times M(C, X) \rightarrow X$ is the evaluation map.

For $f, f^{\prime} \in M(C, X), \bar{k}\left(f, f^{\prime}\right)=0$ is equivalent to $f c=f^{\prime} c$ for all $c \in C$; i.e., $\bar{k}\left(f, f^{\prime}\right)=0$ if and only if $f=f^{\prime}$.

For $\bar{k}\left(f, f^{\prime}\right)<1$, define $\bar{g}\left(\left(f, f^{\prime}\right) \in P M(C, X)\right.$ by

$$
\bar{g}\left(f, f^{\prime}\right)(t)(c)=g\left(f c, f^{\prime} c\right)(t) \text {. }
$$

Letting $U=\bar{k}^{-1}[0,1>$, we conclude that $\bar{g}: U \rightarrow P M(C, X)$ is a morphism from the diagram

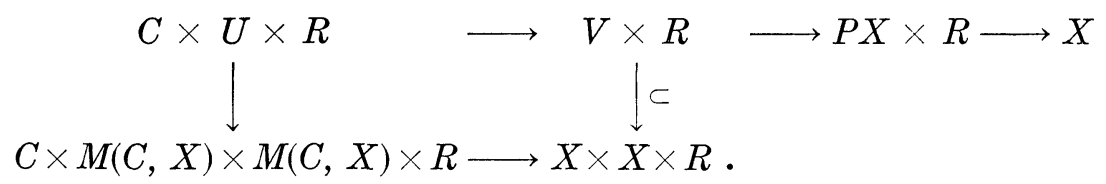

We define $\hat{k}:(C, X, \varphi) \times(C, X, \varphi) \rightarrow I$ to be the restriction of $\bar{k}$ and $\hat{g}: \hat{k}^{-1}[0,1>\rightarrow P(C, X, \varphi)$ to be the restriction of $\bar{g}$. We note that for $f, f^{\prime} \in(C, X, \varphi)$ such that $\bar{k}\left(f, f^{\prime}\right)<1, \bar{g}\left(f, f^{\prime}\right)(t)(a)=g(\varphi a, \varphi a)(t)=$ $\varphi a$. Thus, the restriction of $\bar{g}$ to $\hat{k}^{-1}[0,1>$ factors as asserted in defining $\hat{g}$.

Theorem II.7. If $X$ is LEC and $A$ is a halo retract of $X$, then 
$A$ is LEC and the map $A \rightarrow X$ is a cofibration.

Proof. Let $(k, g)$ be LEC-data for $X, \eta: X \rightarrow I$ be a halo function for $A$, and $r: U \rightarrow A$ be a retraction, where $U=\eta^{-1}[0,1>$.

Define $\psi: X \times X \rightarrow I$ by

$$
\psi(x, y)=\left\{\begin{array}{lll}
\sup _{s \in I}\{\eta(g(x, y) s), k(x, y)\} & \text { for } & k(x, y)<1 \\
1 & \text { for } & k(x, y)=1 .
\end{array}\right.
$$

Then $1-\psi$ is a morphism on Support $(1-k)$ and $1-\psi \leqq 1-k$. Thus, $1-\psi$ and $\psi$ are morphisms. If $\psi(x, y)<1$, the path $g(x, y)$ is defined and lies in $U$. Also, $\psi(x, y)=0$ if and only if $x=y \in A$.

Let $\hat{k}=\left\{\times A\right.$. Then $\hat{k}^{-1} 0$ is the diagonal in $A \times A$. Let $W=\hat{k}^{-1}[0,1>$ and define $\hat{g}: W \rightarrow P A$ by

$$
\hat{g}=(r g, \hat{k}) \text {. }
$$

This new parametrization is a morphism by Lemma 1.5 since the zeroes of $\hat{k}$ are the same as those of $k \mid A \times A$. Thus, $(\hat{k}, \hat{y})$ is LEC-data for $A$.

To verify that $A \rightarrow X$ is a cofibration define $\chi: X \rightarrow 1$ by

$$
\chi(x)= \begin{cases}\sup \{\eta x, k(x, r x)\} & \text { for } \eta x<1 \\ 1 & \text { for } \eta x=1\end{cases}
$$

Exactly as before, $\chi$ is a morphism. $A=\chi^{-1} 0$ and if $\chi x<1$, then $\eta x<1, r x$ is defined and $k(x, r x)<1$. Let $T=\chi^{-1}[0,1>$ and define $h: T \rightarrow P X$ to be the composition

$$
T \stackrel{\Delta}{\longrightarrow} T \times T \stackrel{1_{\mathrm{T}} \times r}{\longrightarrow} V \stackrel{g}{\longrightarrow} P X,
$$

where $V=k^{-1}\left[0,1>\right.$. Then $\chi$ is haloing for $A, T=\chi^{-1}[0,1>$ and $h: T \rightarrow P X$ is a morphism such that $\eta_{0} h=1_{T}, \operatorname{Im}\left(\eta_{-} h\right) \subset A$, and $h \mid A=$ $i_{A}$, where $i_{A}: A \rightarrow P A$ injects each point to the path of zero length at that point. Since for $t \in T, \chi(t)=0$ if and only if $k h t=0$, we can reparametrize the paths $h t$ to have length $\chi(t)$. Then $\chi, T, h$ satisfy condition $(\mathrm{v})$ of the cofibration Theorem I.7.

Corollary II.8. If $X$ is LEC and $x$ is a point of $X$, then the injection $x \rightarrow X$ is a cofibration.

Proof. By the previous theorem, it suffices to show $x$ is a halo retract of $X$. Since a point is a retract of any set containing it, it suffices to show $x$ has a halo in $X$.

Let $k, g$ be LEC-data for $X$. The function $X \times x \rightarrow X \times X$ defined by $\left(x^{\prime}, x\right) \rightarrow\left(x^{\prime}, x\right)$ is a morphism which composed with $k$ defines 
a halo for $x$.

III. The adjunction theorem. J. H. C. Whitehead gave the first proof of this type result for compact, metric ANRs in [7]. Several generalizations have since been made (see [3] and [4]).

Adjunction Theorem. If $X$ and $Y$ are LEC, $A$ is a halo retract in $X$ and $f: A \rightarrow Y$ is a morphism, then any LEC-data for $Y$ can be extended to LEC-data for $X \cup_{f} Y$.

We note this result states not only that $X \mathrm{U}_{f} Y$ is LEC but also relates a LEC structure on it to one on $Y$.

Proof. The proof of this theorem follows from five constructions, stated here. We let $k, g$ be LEC-data for $X$ and $\eta: X \rightarrow I$ be a haloing function for $A$ in $X$.

Step 1. There exists a morphism $\bar{k}: X \times X \rightarrow I$ such that

(i) $\eta x \leqq \bar{k}(x, y)$ and $\eta y \leqq \bar{k}(x, y)$,

(ii) $\triangle A=\bar{k}^{-1} 0$

(iii) for $T=\bar{k}^{-1}\left[0,1>\right.$, the function $G: T \times I^{2} \rightarrow X$ given by

$$
G(x, y)(s, t)=\widehat{g}(\widehat{g}(x, y) s, r \widehat{g}(x, y) s) t
$$

is defined and a morphism, where $\hat{g}$ is the composition $\pi_{1} j g, j$ being the injection $j: P X \rightarrow M(I, X) \times R^{+}$.

Step 2. There exists a morphism $\tilde{k}: X \times X \rightarrow I$ such that

(i) $k \leqq \widetilde{k}$

(ii) $\widetilde{k}^{-1} 0=\Delta X$

(iii) $\bar{k}=1$ and $\tau=0$ implies $\tilde{k}=1$, where $\tau=\inf \left(\eta \pi_{1}, \eta \pi_{2}\right)$.

We note that (ii) implies paths $g$ can be linearly reparametrized by $\widetilde{k}$ to give LEC-data $g$, $\widetilde{k}$ for $X$. Condition (i) implies $g$ is defined on $S=\widetilde{k^{-1}}[0,1>$.

Step 3. In $S$, let

$$
\begin{aligned}
& S_{0}=(\tau \geqq \widetilde{k}) \cup(\tau \geqq 1-\bar{k}) \quad \text { and } \\
& S_{1}=(\tau \leqq \widetilde{k}) \cap(\tau \leqq 1-\bar{k}) .
\end{aligned}
$$

There exists a function $f: S \rightarrow[0,1]$ such that

$$
\begin{aligned}
& f \mid S_{0}=0, \\
& f \mid\left(\tau^{-1} 0-\Delta A\right) \cap S=1, \text { and } \\
& f \mid S-\Delta A \text { is a morphism. }
\end{aligned}
$$


Step 4. There exist morphisms $k_{1}, k_{2}: S \rightarrow[0,1]$ such that

(i) $k_{1}+k_{2}=\tilde{k}$ and $k_{1}=k_{2}$ on $S_{0}$,

(ii) $k_{1}+k_{2} \leqq \tilde{k}$ on $S$, and

(iii) $k_{1}=0$ if and only if $\eta \pi_{1}=0$ or $\tilde{k}=0$ and $k_{2}=0$ if and only if $\eta \pi_{2}=0$ or $\tilde{k}=0$.

Step 5. There exist morphisms $g_{1}, g_{2}: S \rightarrow P X$ and closed sets $M$ and $N$ in $S_{1}$ such that

(i) $l g_{1}=k_{1}, l g_{2}=k_{2}$,

(ii) $g_{1}+g_{2}=g$ on $S_{0}$,

(iii) $\eta_{0} g_{1}=\pi_{1}$ and $\eta_{\tau} g_{2}=\pi_{2}$,

(iv) if $\eta \pi_{1}=0$, then $\eta_{0} g_{2}=r \pi_{2}$ and if $\eta \pi_{2}=0$, then $\eta_{\tau} g_{1}=r \pi_{1}$, and

(v) $S_{0} \cap S_{1} \subset M, \eta_{\tau} g_{1}=\eta_{0} g_{2}$ on $M, \eta_{\tau} g_{1} \in A$ and $\eta_{0} g_{2} \in A$ on $N$, and $\tau^{-1} 0 \subset N$.

Before proving these five assertions, let us prove that the Adjunction Theorem is implied by them.

Extensions of LEC-data $k_{Y}, g_{Y}$ for $Y$ to $X \cup_{f} Y$ will be defined on $(X \Perp Y) \times(X \Perp Y)$ so as to agree under the identifications imposed by the pushout diagram

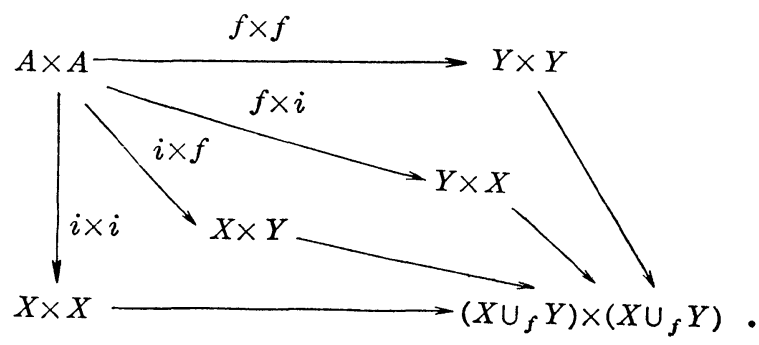

Figure 1

Define $k^{\prime}$ by the following:

on $X \times X$,

$$
k^{\prime}= \begin{cases}\tilde{k} & \text { on } \tilde{k}^{-1} 1 \cup S_{0} \\ k_{1}+k_{2} & \text { on } M \\ k_{1}+k_{Y}\left(f \eta_{-} g_{1}, f \eta_{0} g_{2}\right)+k_{2} & \text { on } \quad N,\end{cases}
$$

on $X \times Y, \quad k^{\prime}=k_{1}\left(\pi_{1}, r \pi_{1}\right)+k_{Y}\left(f r \pi_{1}, \pi_{2}\right)$,

on $Y \times X, \quad k^{\prime}=k_{Y}\left(\pi_{1}, f r \pi_{2}\right)+k_{2}\left(r \pi_{2}, \pi_{2}\right)$, and

on $Y \times Y, \quad k^{\prime}=k_{Y}$.

On the set $k^{\prime-1}\left[0,1>\right.$, define $g^{\prime}$ by: 
on $X \times X$,

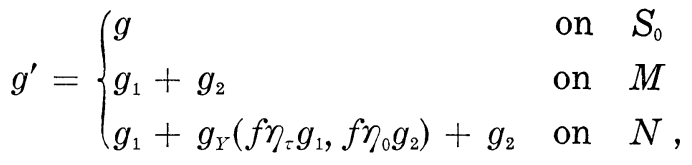

on $X \times Y, \quad g^{\prime}=g_{1}\left(\pi_{1}, r \pi_{1}\right)+g_{Y}\left(f r \pi_{1}, \pi_{2}\right)$,

on $Y \times X, \quad g^{\prime}=g_{Y}\left(\pi_{1}, f r \pi_{2}\right)+g_{2}\left(r \pi_{2}, \pi_{2}\right)$, and

on $Y \times X, \quad g^{\prime}=g_{Y}$.

Checking the conditions as stated in Steps 1 through 5 when relevant shows the functions as defined are morphisms, agree as required on $A \times A$, and define LEC-data on $X \mathrm{U}_{f} Y$.

We next establish the constructions asserted in Steps 1 through 5 .

Step 1. Let $k, g$ be LEC-data for $X, \eta$ a halo for $A$ in $X$ and $r: U \rightarrow A$ a retraction, $U=\eta^{-1}[0,1>$.

Define $\hat{k}: X \times X \rightarrow I$ by

$$
\hat{k}(x, y)=\left\{\begin{array}{lll}
\sup _{s \in I}\{k(x, y), \eta g(x, y) s\} & \text { for } k(x, y)<1 \\
1 & \text { for } k(x, y)=1 .
\end{array}\right.
$$

The $1-\hat{k} \leqq 1-k$ and $1-\hat{k}$ is a morphism on the support of $1-k$. Thus, $\hat{k}$ is a morphism.

Define $\breve{k}: X \times X \rightarrow I$ by

$$
\breve{k}(x, y)= \begin{cases}\sup _{s \in I}\{(\hat{k}(x, y), k(g(x, y) s, r g(x, y) s)\} & \text { for } \hat{k}(x, y)<1 \\ 1 & \text { for } \hat{k}(x, y)=1 .\end{cases}
$$

As above, $\check{k}$ is a morphism.

Finally, define $\bar{k}: X \times X \rightarrow I$ by

$$
\begin{aligned}
\bar{k}(x, y)=\sup _{s, s^{\prime}, t, t^{\prime} \in I^{4}}\{\check{k}(x, y), k(g(g(x, y) s, r g(x, y) s) t, \\
\\
\left.\left.g\left(g(x, y) s^{\prime}, r g(x, y) s^{\prime}\right) t^{\prime}\right)\right\}
\end{aligned}
$$

for $\breve{k}<1$ and $\bar{k}(x, y)=1$ for $\hat{k}(x, y)=1$. As before $\bar{k}$ is a morphism.

Observe that $\eta x \leqq \bar{k}(x, y)$ and $\eta y \leqq \bar{k}(x, y)$; also, $\bar{k}^{-1} 0=\Delta A$. The conditions imposed by $\bar{k}$ being less than 1 are sufficient to establish $G$ has the asserted properties.

Step 2. Let $\tilde{k}=\sup \{k, k / 1+\tau+k-\bar{k}\}$. The denominator $1+$ $\tau+k-\bar{k} \neq 0$ since $\bar{k}=1$ and $\tau=0$ imply $k>0$. Thus, $\widetilde{k}$ is a morphism. By definition, $k \leqq \widetilde{k}$. Also, $\widetilde{k}=0$ if and only if $k=0$; and so, $\widetilde{k}^{-1} 0=\Delta X$. Clearly, for $\bar{k}=1$ and $\tau=0, \widetilde{k}=1$. 
Step 3. For $S=\widetilde{k}^{-1}[0,1>$, let

$$
\begin{aligned}
& S_{0}=\{(\tau \geqq \widetilde{k}) \cup(\tau \geqq 1-\bar{k})\} \cap S \text { and } \\
& S_{1}=\{(\tau \leqq \widetilde{k}) \cap(\tau \leqq 1-\bar{k})\} \cap S .
\end{aligned}
$$

Define the function $d: S \rightarrow I$ by $d \mid S_{0}=0$ (note that $\Delta A \subset S_{0}$ )

$$
d=(\widetilde{k}-\tau)(1-\bar{k}-\tau) / \widetilde{k} \cdot(1-\bar{k}) \quad \text { on } \quad S_{1}-\Delta A .
$$

The two definitions agree on $\left(S_{0}-\Delta A\right) \cap\left(S_{1}-\Delta A\right)$. These two sets are closed in $S-\Delta A$. To check that $d \mid S-\Delta A$ is a morphism it suffices to check that $d \mid S_{1}-\Delta A$ is one.

Since $\widetilde{k}<1$, either $\bar{k}<1$ or $\tau>0$. In $S_{1}, \bar{k}=1$ implies $\tau=0$. Thus, $\bar{k}<1$. If $\tilde{k}=0$, then $\tau=0$, and the point is in $\Delta A$. Hence, in $S_{1}-\Delta A, \widetilde{k} \cdot(1-\bar{k})>0$; and so, $d \mid S_{1}-\Delta A$ is a morphism.

Since $\left(\tau^{-1} 0-\Delta A\right) \cap S \subset S_{1}-\Delta, d \mid\left(\tau^{-1} 0-\Delta A\right) \cap S=1$.

Step 4. Define $\bar{k}: S \rightarrow I$ by

$$
\bar{k}= \begin{cases}\inf \left\{\tilde{k},(1-d) \cdot \widetilde{k}+d \cdot\left(\eta \pi_{1}+\eta \pi_{2}\right)\right\} & \text { on Support } \tilde{k} \\ 0 & \text { on } \tilde{k}^{-1} 0 .\end{cases}
$$

Then $\vec{k} \leqq \widetilde{k}$ and $\vec{k}$ is a morphism on Support $\widetilde{k}$. Thus $\vec{k}$ is a morphim on $S$.

Let $\hat{\varphi}=\inf \left(1, \eta \pi_{1}+\eta \pi_{2}\right)$ and define $k_{1}^{\prime}: S \rightarrow I$ by

$$
k_{1}^{\prime}= \begin{cases}\bar{k} \cdot \eta \pi_{1} / \hat{\varphi} & \text { for } \hat{\varphi}>0 \\ 0 & \text { for } \hat{\varphi}=0 .\end{cases}
$$

That $k_{1}^{\prime}$ is a morphism follows from Lemma I.2 by the following argument:

$$
\begin{aligned}
& \hat{\varphi}: X \times X \longrightarrow I \text { is a morphism, } \\
& \eta=\hat{\psi}: X \longrightarrow I \text { is a morphism, and } \\
& \hat{h}: X \times X \times I \longrightarrow I \text { by } \hat{h}=\left(\hat{k} \circ\left(\rho_{1} \times \rho_{2}\right)\right) \cdot \rho_{3},
\end{aligned}
$$

the $\rho_{i}$ 's being projections, is a morphism. If $\hat{\varphi}=0$, then $\tilde{k}=0$ or $d=1$. In either case $\vec{k}=0$; and so, $\hat{h}$ is independent of $t \in I$ if $\hat{\varphi}=$ 0 .

Let $W \subset X \times X \times X \times I$ be

$$
W=\left\{\left(x_{1}, x_{2}\right), x, t \mid t \cdot \hat{\psi} x \leqq \hat{\varphi}\left(x_{1}, x_{2}\right)\right\} .
$$

By Lemma I.2 $c: W \rightarrow I$ defined by

$$
c\left(\left(x_{1}, x_{2}\right),(x, t)= \begin{cases}\bar{k}\left(x_{1}, x_{2}\right) \cdot t \cdot \eta x / \hat{\varphi}\left(x_{1}, x_{2}\right) & \text { for } \hat{\varphi}>0 \\ 0 & \text { for } \hat{\varphi}=0\end{cases}\right.
$$


is a morphism. Define $j: S \rightarrow W$ by $j\left(x_{1}, x_{2}\right)=\left(\left(x_{1}, x_{2}\right), x_{1}, 1\right)$. Then

$$
\begin{aligned}
c \cdot j\left(x_{1}, x_{2}\right) & = \begin{cases}\vec{k}\left(x_{1}, x_{2}\right) \cdot \eta x_{1} / \widehat{\varphi}\left(x_{1}, x_{2}\right) & \text { for } \hat{\varphi}\left(x_{1}, x_{2}\right) \neq 0 \\
0 & \text { for } \hat{\varphi}\left(x_{1}, x_{2}\right)=0\end{cases} \\
& =k_{1}^{\prime}
\end{aligned}
$$

is a morphism on $S$.

Define $\gamma: X \times X \rightarrow I$ by

$$
\gamma= \begin{cases}1 / \eta \pi_{1}+\eta \pi_{2} & \text { for } 1 \leqq \eta \pi_{1}+\eta \pi_{2} \\ 1 & \text { for } \eta \pi_{1}+\eta \pi_{2} \leqq 1\end{cases}
$$

Since $\gamma$ is a morphism, so is $\widetilde{k}_{1}=k_{1}^{\prime} \cdot \gamma$. Thus,

$$
\tilde{k}_{1}= \begin{cases}\vec{k} \cdot \eta \pi_{1} / \eta \pi_{1}+\eta \pi_{2} & \text { for } 0<\eta \pi_{1}+\eta \pi_{2} \\ 0 & \text { for } 0=\eta \pi_{1}+\eta \pi_{2}\end{cases}
$$

and

$$
\widetilde{k}_{2}= \begin{cases}\bar{k} \cdot \eta \pi_{2} / \eta \pi_{1}+\eta \pi_{2} & \text { for } 0<\eta \pi_{1}+\eta \pi_{2} \\ 0 & \text { for } 0=\eta \pi_{1}+\eta \pi_{2}\end{cases}
$$

are morphisms on $S$. Clearly, $\widetilde{k}_{1}+\widetilde{k}_{2}=\vec{k}$ where $0<\eta \pi_{1}+\eta \pi_{2}$. If $0=\eta \pi_{1}+\eta \pi_{2}$, then either $\widetilde{k}=0$ or $\tau=0$ and $d=1$. In either case $\vec{k}=0$. Thus, $\widetilde{k}_{1}+\widetilde{k}_{2}=\widetilde{k}$ on $S$; and so, $\widetilde{k}_{1}+\widetilde{k}_{2}=\vec{k} \leqq \tilde{k}$ for all $(x, y) \in$ S. For $(x, y) \in S_{0}, d=0$ and $\tilde{k}=\widetilde{k}$; thus, on $S_{0} \widetilde{k}_{1}+\widetilde{k}_{2}=\widetilde{k} . \quad \widetilde{k}_{1}=0$ implies $\vec{k}=0$ or $\eta \pi_{1}=0$, which implies $\widetilde{k}=0$ or $\eta \pi_{1}=0$, which implies $\vec{k}=0$ or $\eta \pi_{1}=0$, which implies $\widetilde{k}_{1}=0$. Thus, $\widetilde{k}_{1}=0$ if and only if $\widetilde{k}=0$ or $\eta \pi_{1}=0$. Similarly, $\widetilde{k}_{2}=0$ if and only if $\widetilde{k}=0$ or $\eta \pi_{2}=0$. Let $k_{1}=\left(\widetilde{k}_{1}-(1 / 2) \widetilde{k}\right) \cdot d+(1 / 2) \widetilde{k}$ and $k_{2}=\left(\widetilde{k}_{2}-(1 / 2) \widetilde{k}\right) \cdot d+(1 / 2) \widetilde{k}$. Since $k_{i}, i=1,2$, is a morphism on Support $2 \widetilde{k}$ and $k_{i} \leqq 2 \widetilde{k}, k_{i}$ is a morphism on $S$. Also, $0 \leqq k_{i} \leqq 1$. Furthermore,

$$
k_{1}+k_{2}=\left(\widetilde{k}_{1}+\widetilde{k}_{2}-\tilde{k}\right) \cdot d+\widetilde{k} \leqq \tilde{k} .
$$

On $S_{0}, d=0$ and so, $k_{1}=k_{2}=(1 / 2) \tilde{k}$. Finally, $k_{1}=0$ if and only if $\widetilde{k}_{1} d=((1 / 2) d-1 / 2) \tilde{k}$. But the latter is true if and only if $\widetilde{k}_{1}=0$ and $\widetilde{k}=0$ or $\widetilde{k}_{1}=0$ and $d=1$ or $d=0$ and $\widetilde{k}=0$. This is true if and only if $\widetilde{k}_{1}=0$. Thus, $k_{1}=0$ if and only if $\eta \pi_{1}=0$ or $\widetilde{k}=0$ and $k_{2}=$ 0 if and only if $\eta \pi_{2}=0$ or $\widetilde{k}=0$.

Step 5. Let $M=d^{-1}[0,2 / \pi \arctan 2] \cap S_{1}$ and

$$
N=\left\{y^{-1}[2 / \pi \arctan 2,1] \cap S_{1}\right\} \cup \Delta A .
$$

Then $S_{0} \cap S_{1} \subset M$ and $S_{0} \cap N=\Delta A$. Define $a$ and $b$ mapping $I$ in $P I^{2}$ by 


$$
a(t) s= \begin{cases}\left(\frac{1}{2} s, \frac{1}{2} s \tan \pi t / 2\right) & \text { for } \quad 0 \leqq \pi t / 2 \leqq \arctan 2 \\ (s \cot \pi t / 2, s) & \text { for } \quad \arctan 2 \leqq \pi t / 2 \leqq \pi / 2\end{cases}
$$

and

$$
b(t) s=\left\{\begin{array}{l}
\left(\frac{1}{2}+\frac{1}{2} s,\left(\frac{1}{2}-\frac{1}{2} s\right) \tan \pi t / 2\right) 0 \leqq \pi t / 2 \leqq \arctan 2 \\
(1+(s-1) \cot \pi t / 2,1-s) \arctan 2 \leqq \pi t / 2 \leqq \pi / 2 .
\end{array}\right.
$$

The following assertions are easily verified:

(1) for $0 \leqq \pi t / 2 \leqq \arctan 2, a(t) 1=b(t) 0$,

(2) for $\operatorname{arc} \tan 2 \leqq \pi t / 2 \leqq 1, \pi_{2} a(t) 1=1=\pi_{2} b(t) 0$, and

(3) $a(t)(0)=(0,0)$ and $b(t)(1)=(1,0)$ for all $t$.

Define $g_{1}: S \rightarrow P X$ by

$$
g_{1}\left(x, x^{\prime}\right)=\left\{\begin{array}{lll}
\left.g\left(x, x^{\prime}\right)\right|_{0} ^{k_{1}\left(x, x^{\prime}\right)} & \text { with length } k_{1} \text { for } & \left(x, x^{\prime}\right) \in S_{0} \\
G\left(x, x^{\prime}\right) \mid \alpha \circ d\left(x, x^{\prime}\right) & \text { with length } k_{1} \text { for } \quad\left(x, x^{\prime}\right) \in S_{1} .
\end{array}\right.
$$

On $S_{0} \cap S_{1}, \tau=\widetilde{k}=1-\bar{k}, d=0$ and $k_{1}=(1 / 2) \tilde{k} . \quad a \circ d$ is the interval $[(0,0),(1 / 2,0)]$. The composition

$$
\begin{aligned}
& G\left(x, x^{\prime}\right) \mid a \circ d=\hat{g}\left(\widehat{g}\left(x, x^{\prime}\right) s, r \widehat{g}\left(x, x^{\prime}\right) s\right) 0, \quad 0 \leqq s \leqq \frac{1}{2} \\
& =\widehat{g}\left(x, x^{\prime}\right) s \\
& 0 \leqq s \leqq \frac{1}{2} \\
& =\left.\hat{g}\left(x, x^{\prime}\right)\right|_{0} ^{1 / 2} \\
& =\left.\pi_{1} j g\left(x, x^{\prime}\right)\right|_{0} ^{(1 / 2) \tilde{k}\left(x, x^{\prime}\right)} \\
& =\left.\pi_{1} j g\left(x, x^{\prime}\right)\right|_{0} ^{k_{1}\left(x, x^{\prime}\right)} \text {. }
\end{aligned}
$$

Thus, the definitions of $g_{1}$ on $S_{0}$ and $S_{1}$ agree on $S_{0} \cap S_{1}$. Clearly, $g_{1} \mid S_{0}$ is a morphism. To check that $g_{1} \mid S_{1}$ is also, it suffices to check $\pi_{1} j g \mid S_{1}$. On $S_{1}, \bar{k}<1$. Define a morphism $h: T \times M\left(I, I^{2}\right) \times I \rightarrow X$ by the following function space adjointness applications:

$$
\begin{aligned}
G: & T \times I^{2} \longrightarrow X, \\
\hat{G}: I^{2} \longrightarrow M(T, X), & \longrightarrow M(I, M(T, X)) \cong M(T, M(I, X)), \\
M(I, \widehat{G}): & M\left(I, I^{2}\right) \longrightarrow M(I, X), \quad \text { and finally } \\
M(I, \widehat{G})^{\sim}: & T \times M\left(I, I^{2}\right) \longrightarrow M\left(I, I^{2}\right) \times I \longrightarrow X .
\end{aligned}
$$

Define $\rho: T \rightarrow M\left(I, I^{2}\right)$ by

$$
\rho\left(x, x^{\prime}\right)=\left\{\begin{array}{lll}
a d\left(x, x^{\prime}\right) & \text { if } & \bar{k}\left(x, x^{\prime}\right)>0 \\
[0,0),(0,1)] & \text { if } & \bar{k}\left(x^{\prime}, x\right)=0
\end{array}\right.
$$

Then $\bar{k}, h, \rho$ satisfy the hypotheses of Lemma I.2. Thus, 


$$
k\left(x, x^{\prime}, t\right)=h\left(x, x^{\prime}, \rho\left(x, x^{\prime}\right), t\right)
$$

is a morphism. But this is $\pi_{1} j g_{1} \mid S_{1}$.

Similarly,

$$
g_{2}\left(x, x^{\prime}\right)= \begin{cases}g\left(x, x^{\prime}\right) \mid \begin{array}{l}
\tilde{k}\left(x, x^{\prime}\right) \\
k_{1}\left(x, x^{\prime}\right)
\end{array} & \text { with length } k_{2}\left(x, x^{\prime}\right) \text { on } S_{0} \\
G\left(x, x^{\prime}\right) \mid b d\left(x, x^{\prime}\right) & \text { with length } k_{2}\left(x, x^{\prime}\right) \text { on } S_{1}\end{cases}
$$

is a morphism.

Verification of ( $\mathrm{i}$ ) and (ii) of Step 5 is immediate. Condition ( v) follows from (1) and (2) above and condition (iii) follows from (3). To check condition (iv), observe that $\eta \pi_{1}=0$ implies $d=1$ or $\bar{k}=0$. In the latter case $g_{2}$ is the 0 -path at $x=x^{\prime}=r x=r x^{\prime}$. In the former,

$$
\pi_{1} j g_{2} s=\left(\widehat{g}\left(\widehat{g}\left(x, x^{\prime}\right) 1, r \widehat{g}\left(x, x^{\prime}\right) 1\right)(1-s) \quad 0 \leqq s \leqq 1 .\right.
$$

and so,

$$
\eta_{0} g_{2}=\pi_{1} j g_{2} 0=r x^{\prime}=r \pi_{2}
$$

CoRollary III.2. Every cell-complex is LEC.

Proof. A cell-complex $X$ is the colimit of a sequence of morphisms $X^{(n)} \stackrel{f_{n}}{\longrightarrow} X^{(n+1)}$, where $X^{0}$ is a discrete space and for each $n, f_{n}$ is defined by the push-out diagram

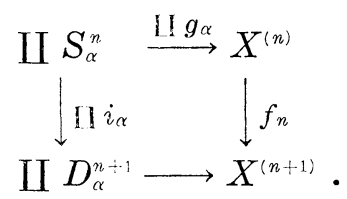

II $D_{\alpha}^{n+1}$ is LEC and as II $i_{\alpha}$ is a cofibration, II $S_{\alpha}^{n}$ is a halo retract in II $D_{\alpha}^{n+1}$. Thus, by the Adjunction Theorem LEC-data for $X^{(n)}$ extends to LEC-data for $X^{(n+1)}$. $X^{(0)}$, being discrete, is LEC. Inductively, a sequence $\left\{g^{(n)}, k^{(n)}\right\}$ of LEC-data is formed for the $\left\{X^{(n)}\right\}$ such that each extends its predecessor. The functions $g, k$ defined on the colimit $X$ are thus morphisms and are LEC-data for $X$.

\section{REFERENCES}

1. J. Dugundji, Locally equiconnected spaces and absolute neighborhood retracts, Fund. Math., 55 (1965), 187-193.

2. - Topology, Allyn and Bacon, Boston, 1966.

3. D. M. Hyman, A category slightly larger than the metric and CW-categories, Michigan Math. J., 15 (1968), 193-214.

4. W. D. McIntosh, Adjunction of locally equiconnected spaces, Fund. Math., 63 (1968), 175-192.

5. E. H. Spanier, Quasi-Topologies, Duke Math. J., 30 (1963). 1-14. 
6. N. E. Steenrod, A convenient category of topological spaces, Michigan Math. J., 14 (1967), 133-152.

7. J. H. C. Whitehead, Note on a theorem due to Borsuk, Bull. Amer. Math. Soc., 54 (1948), 1125-1132.

8. G. S. Young, $A$ condition for the absolute homotopy extension property, Amer. Math. Monthly, 71 (1964), 896-897.

Received February 5, 1971 and in revised form May 19, 1971.

Cuny Graduate Center

AND

Columbia University 



\title{
PACIFIC JOURNAL OF MATHEMATICS
}

\author{
EDITORS
}

H. SAMELSON

Stanford University

Stanford, California 94305

C. R. HOBBY

University of Washington

Seattle, Washington 98105

\section{J. DugundJI}

Department of Mathematics

University of Southern California

Los Angeles, California 90007

RICHARD ARENS

University of California

Los Angeles, California 90024

\section{ASSOCIATE EDITORS}
E. F. BeCKeNBACH
B. H. NeUmanN
F. WoLF
K. YoSHIDA

\section{SUPPORTING INSTITUTIONS}

\author{
UNIVERSITY OF BRITISH COLUMBIA \\ CALIFORNIA INSTITUTE OF TECHNOLOGY \\ UNIVERSITY OF CALIFORNIA \\ MONTANA STATE UNIVERSITY \\ UNIVERSITY OF NEVADA \\ NEW MEXICO STATE UNIVERSITY \\ OREGON STATE UNIVERSITY \\ UNIVERSITY OF OREGON \\ OSAKA UNIVERSITY
}

\author{
UNIVERSITY OF SOUTHERN CALIFORNIA \\ STANFORD UNIVERSITY \\ UNIVERSITY OF TOKYO \\ UNIVERSITY OF UTAH \\ WASHINGTON STATE UNIVERSITY \\ UNIVERSITY OF WASHINGTON

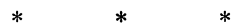 \\ AMERICAN MATHEMATICAL SOCIETY \\ NAVAL WEAPONS CENTER
}

The Supporting Institutions listed above contribute to the cost of publication of this Journal, but they are not owners or publishers and have no responsibility for its content or policies.

Mathematical papers intended for publication in the Pacific Journal of Mathematics should be in typed form or offset-reproduced, (not dittoed), double spaced with large margins. Underline Greek letters in red, German in green, and script in blue. The first paragraph or two must be capable of being used separately as a synopsis of the entire paper. The editorial "we" must not be used in the synopsis, and items of the bibliography should not be cited there unless absolutely necessary, in which case they must be identified by author and Journal, rather than by item number. Manuscripts, in duplicate if possible, may be sent to any one of the four editors. Please classify according to the scheme of Math. Rev. Index to Vol. 39. All other communications to the editors should be addressed to the managing editor, Richard Arens, University of California, Los Angeles, California, 90024.

50 reprints are provided free for each article; additional copies may be obtained at cost in multiples of 50 .

The Pacific Journal of Mathematics is published monthly. Effective with Volume 16 the price per volume (3 numbers) is $\$ 8.00$; single issues, $\$ 3.00$. Special price for current issues to individual faculty members of supporting institutions and to individual members of the American Mathematical Society: $\$ 4.00$ per volume; single issues $\$ 1.50$. Back numbers are available.

Subscriptions, orders for back numbers, and changes of address should be sent to Pacific Journal of Mathematics, 103 Highland Boulevard, Berkeley, California, 94708.

PUBLISHED BY PACIFIC JOURNAL OF MATHEMATICS, A NON-PROFIT CORPORATION

Printed at Kokusai Bunken Insatsusha (International Academic Printing Co., Ltd.), 270, 3-chome Totsuka-cho, Shinjuku-ku, Tokyo 160, Japan. 


\section{Pacific Journal of Mathematics}

\section{Vol. 41, No. $3 \quad$ BadMonth, 1972}

George E. Andrews, Two theorems of Gauss and allied identities proved arithmetically.................................. 563

Stefan Bergman, On pseudo-conformal mappings of circular domains . . . . 579

Beverly L. Brechner, On the non-monotony of dimension ............ 587

Richard Anthony Brualdi and John H. Mason, Transversal matroids and Hall's theorem .................................... 601

Philip Throop Church and James Timourian, Differentiable maps with

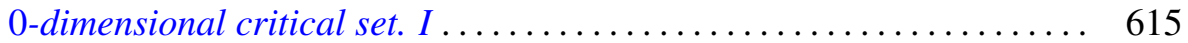

John H. E. Cohn, Squares in some recurrent sequences ............. 631

Robert S. Cunningham, Edgar Andrews Rutter and Darrell R. Turnidge, Rings of quotients of endomorphism rings of projective modules ......

Eldon Dyer and S. Eilenberg, An adjunction theorem for locally equiconnected spaces................................... 669

Michael W. Evans, On commutative P. P. rings................. 687

Ronald Lewis Graham, Hans Sylvain Witsenhausen and Hans Zassenhaus, On tightest packings in the Minkowski plane ..................

Stanley P. Gudder, Partial algebraic structures associated with

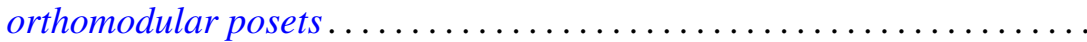

Karl Edwin Gustafson and Gunter Lumer, Multiplicative perturbation of

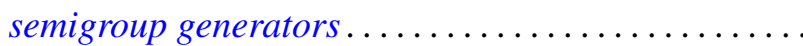

Kurt Kreith and Curtis Clyde Travis, Jr., Oscillation criteria for selfadjoint elliptic equations...

Lawrence Louis Larmore, Twisted cohomology theories and the single obstruction to lifting...........................

Jorge Martinez, Tensor products of partially ordered groups . . .

Robert Alan Morris, The inflation-restriction theorem for Amitsur cohomology ...

Leo Sario and Cecilia Wang, The class of $(p, q)$-biharmonic functions ...

Manda Butchi Suryanarayana, On multidimensional integral equations of

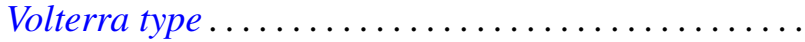

